\title{
Pathology parameters and adjuvant tamoxifen response in a randomised premenopausal breast cancer trial
}

\author{
K Jirström, L Rydén, L Anagnostaki, B Nordenskiöld, O Stål, S Thorstenson, G Chebil, P-E Jönsson, \\ M Fernö, G Landberg
}

J Clin Pathol 2005;58:1135-1142. doi: 10.1136/jcp.2005.027185

See end of article for authors' affiliations .............

Correspondence to: Dr K Jirström, Division of Pathology, Department of Laboratory Medicine, Lund University, Malmö University Hospital, SE205 02, Malmö, Sweden; karin.jirstrom@pat.mas.lu. se

Accepted for publication 29 March 2005
Background: Subgroups of breast cancer that have an impaired response to endocrine treatment, despite hormone receptor positivity, are still poorly defined. Breast cancer can be subdivided according to standard pathological parameters including histological type, grade, and assessment of proliferation. These parameters are the net result of combinations of genetic alterations effecting tumour behaviour and could potentially reflect subtypes that respond differently to endocrine treatment.

Aims: To investigate the usefulness of these parameters as predictors of the response to tamoxifen in premenopausal women with breast cancer.

Materials/methods: Clinically established pathological parameters were assessed and related to the tamoxifen response in 500 available tumour specimens from 564 premenopausal patients with breast cancer randomised to either two years of tamoxifen or no treatment with 14 years of follow up. Proliferation was further evaluated by immunohistochemical Ki-67 expression.

Results: Oestrogen receptor positive ductal carcinomas responded as expected to tamoxifen, whereas the difference in recurrence free survival between control and tamoxifen treated patients was less apparent in the relatively few lobular carcinomas. For histological grade, there was no obvious difference in treatment response between the groups. The relation between proliferation and tamoxifen response seemed to be more complex, with a clear response in tumours with high and low proliferation, whereas tumours with intermediate proliferation defined by $\mathrm{Ki}-67$ responded more poorly.

Conclusions: Clinically established pathology parameters seem to mirror the endocrine treatment response and could potentially be valuable in future treatment decisions for patients with breast cancer.
$\mathrm{P}$ atients with breast cancer who have hormone receptor positive tumours benefit from adjuvant tamoxifen treatment, regardless of nodal status, menopausal status, and age. ${ }^{1}$ Both recurrence free survival (RFS) and breast cancer survival (BCS) are improved and prolonged treatment (five years) enhances the effect. ${ }^{1-5}$ Hormone receptor positivity is fundamental for the response to tamoxifen; oestrogen receptor (ER) status is the best established predictive marker, ${ }^{16}$ and progesterone receptor (PR) status seems to be an important alternative predictor. ${ }^{67}$

Nevertheless, not all patients with breast cancer who have steroid receptor positive tumours respond to antioestrogen treatment as a result of either de novo or acquired resistance. New predictive markers for tamoxifen response are continually being investigated; HER2 is one of the best studied of these markers and is also important because of the availability of targeted treatment against this receptor. ${ }^{8-10}$ Modern genomic and proteomic screening techniques in combination with high throughput tissue analyses/tissue microarrays have facilitated the process of identifying and evaluating candidate disease genes responsible for the clinical behaviour of tumours. Nevertheless, the different phenotypes are reflected by variations in standard clinicopathological parameters, such as histological type, histological grade, and proliferative activity. These parameters are readily assessed and constitute well established prognostic factors, although their influence upon the response to endocrine treatment needs further investigation. The beneficial effect of tamoxifen has been demonstrated in tumours of all histological grades, ${ }^{11}$ whereas there are few reports on the relation between histological type and endocrine response. In one retrospective study of 267 invasive ductal carcinomas (IDCs) and 33 invasive lobular carcinomas (ILCs), no difference in endocrine response between the two groups was seen, despite almost all ILCs being hormone receptor positive. ${ }^{12}$ Another, more recent study, found that ILC had a worse response to neoadjuvant chemotherapy. ${ }^{13}$

"Not all patients with breast cancer who have steroid receptor positive tumours respond to antioestrogen treatment as a result of either de novo or acquired resistance ${ }^{\prime \prime}$

The growth kinetics of breast tumours have a clear relation with clinical outcome, and most studies using proliferation markers have shown that they have prognostic significance ${ }^{14-18}$ and predictive value for response to adjuvant chemotherapy ${ }^{19-23}$ Only a few studies have investigated the usefulness of proliferation markers for predicting response to endocrine treatment and the results are somewhat conflicting, most indicating a better response in tumours with a low proliferation rate. $^{24-27}$ Therefore, the aim of our present study was to investigate the response to adjuvant tamoxifen in relation to histological type, grade, and proliferative activity—as assessed by mitotic index (MI) and Ki-67 index-in premenopausal patients included in a randomised trial.

Abbreviations: $B C S$, breast cancer survival; ER, oestrogen receptor; IDC, invasive ductal carcinomas; ILC, invasive lobular carcinoma; MI, mitotic index; NHG, Nottingham histological grade; PR, progesterone receptor; RFS, recurrence free survival 
Table 1 Clinicopathological characteristics in ductal and lobular carcinomas

\begin{tabular}{|c|c|c|c|}
\hline & $\begin{array}{l}\text { IDC } \\
n=411 \quad(\%)\end{array}$ & $\begin{array}{l}\text { ILC } \\
n=43(\%)\end{array}$ & p Value \\
\hline Randomisation & & & 0.99 \\
\hline Tamoxifen & $201(48.9)$ & $21(48.8)$ & \\
\hline Control & $210(51.1)$ & $22(51.2)$ & \\
\hline Age & & & 0.49 \\
\hline$<40$ years & $77(18.7)$ & $5(11.6)$ & \\
\hline $40-49$ years & 271 (65.9) & $30(69.8)$ & \\
\hline $50-59$ years & $63(15.3)$ & $8(18)$ & \\
\hline Tumour size & & & 0.47 \\
\hline $0-10 \mathrm{~mm}$ & $24(5.8)$ & $3(6.9)$ & \\
\hline $11-20 \mathrm{~mm}$ & $133(32.4)$ & $10(23.2)$ & \\
\hline $21+\mathrm{mm}$ & $253(61.5)$ & $30(69.8)$ & \\
\hline Not registered & $1(0.2)$ & & \\
\hline Lymph node status & & & 0.88 \\
\hline 0 & $103(25.0)$ & $12(27.9)$ & \\
\hline $1-3$ & $212(51.6)$ & $20(46.5)$ & \\
\hline 4 & $94(22.9)$ & $11(25.6)$ & \\
\hline Not registered & $2(0.5)$ & & \\
\hline Histological grade & & & $<0.0001$ \\
\hline 1 & $47(11.4)$ & $1(2.3)$ & \\
\hline$\|$ & $170(41.3)$ & $34(79.1)$ & \\
\hline III & $182(44.3)$ & $7(16.3)$ & \\
\hline Not evaluated & $12(2.9)$ & $1(2.3)$ & \\
\hline Mitotic index & & & $<0.0001$ \\
\hline 1 & $120(29.2)$ & $30(69.7)$ & \\
\hline 2 & $144(35.5)$ & $8(18.6)$ & \\
\hline 3 & $135(32.8)$ & $4(9.3)$ & \\
\hline Not evaluated & $12(2.9)$ & $1(2.3)$ & \\
\hline $\mathrm{Ki}-67$ index & & & 0.001 \\
\hline $0-10 \%$ & 161 (39.2) & $25(58.1)$ & \\
\hline $11-25 \%$ & $105(25.5)$ & $5(11.6)$ & \\
\hline$>25 \%$ & $93(25.5)$ & $2(4.6)$ & \\
\hline Not evaluated & $52(12.6)$ & $11(25.6)$ & \\
\hline ER status & & & 0.01 \\
\hline Negative & $122(29.7)$ & $4(9.3)$ & \\
\hline Positive & $275(66.9)$ & $33(76.7)$ & \\
\hline Not evaluated & $14(3.4)$ & $5(11.6)$ & \\
\hline PR status & & & 0.01 \\
\hline Negative & 118 (28.7) & $4(9.3)$ & \\
\hline Positive & $263(64.0)$ & $33(76.7)$ & \\
\hline Not evaluated & $30(7.2)$ & $6(13.9)$ & \\
\hline \multicolumn{4}{|c|}{$\begin{array}{l}\text { The } p \text { values were assessed by the } \chi^{2} \text { test. } \\
\text { ER, oestrogen receptor; IDC, invasive ductal carcinomas; ILC, invasive } \\
\text { lobular carcinoma; PR, progesterone receptor. }\end{array}$} \\
\hline
\end{tabular}

\section{MATERIALS AND METHODS \\ Patient material}

Between 1986 and 1991, 564 premenopausal patients or patients under 50 years of age with stage II (pT2 N0 M0, pTl $\mathrm{Nl} \mathrm{M} 0$, and pT2 Nl M0) invasive breast cancer were enrolled in a randomised trial of adjuvant tamoxifen treatment. The patients were staged according to the International Union against Cancer TNM classification. ${ }^{28}$ Fifty seven of 276 patients in the tamoxifen treated arm and seventy of 288 in the control arm had four or more positive lymph nodes. Patients were included irrespective of hormone receptor status. The daily dosage of tamoxifen used was either $40 \mathrm{mg}$ (study center 1) or $20 \mathrm{mg}$ (study center 2). Similar results for these dosages have been found in postmenopausal patients. ${ }^{5}{ }^{10}$ Patients were considered premenopausal until one year after last menstruation. Patient records were reevaluated for patients older than 50 years at inclusion to ensure that they were premenopausal at the time of randomisation. All patients underwent modified radical mastectomy or breast conserving surgery with axillary lymph node dissection. In the last group, the breast was treated with radiotherapy, in addition to locoregional radiotherapy for those patients with axillary lymph node metastases. Fewer than $2 \%$ of the patients $(n=9)$ received adjuvant polychemotherapy.

The median follow up for patients without a breast cancer event was 13.9 years (95\% confidence interval, 13.6 to 14.3 ) and it did not differ between the two treatment arms. Clinical and tumour characteristics were also well balanced in the two study groups. The study is described in detail elsewhere. ${ }^{29}$ All tumours had previously been histopathologically reevaluated and graded according to Elston and Ellis ${ }^{30}$ in 491 cases.

Table 3 Clinicopathological characteristics in relation to $\mathrm{Ki}-67$ index

\begin{tabular}{|c|c|c|c|c|}
\hline & \multicolumn{3}{|l|}{ Ki-67 index } & \multirow[b]{2}{*}{ p Value } \\
\hline & Low & Intermediate & High & \\
\hline Total number & $194(\%)$ & $118(\%)$ & $118(\%)$ & \\
\hline Randomisation & & & & 0.12 \\
\hline Tamoxifen & $85(43.8)$ & $66(55.9)$ & $57(48.3)$ & \\
\hline Control & $109(56.2)$ & $52(44.1)$ & $61(51.7)$ & \\
\hline Age & & & & 0.38 \\
\hline$<40$ years & $32(16.5)$ & $24(20.3)$ & $26(22.0)$ & \\
\hline $40-49$ years & $126(64.9)$ & $79(66.9)$ & $78(66.1)$ & \\
\hline $50-59$ years & $36(18.6)$ & 1512.71 & $14(11.8)$ & \\
\hline Tumour size & & & & 0.01 \\
\hline $0-10 \mathrm{~mm}$ & $14(7.2)$ & $8(6.8)$ & $6(5.1)$ & \\
\hline $11-20 \mathrm{~mm}$ & $68(35.0)$ & $35(29.7)$ & $21(17.8)$ & \\
\hline $21+\mathrm{mm}$ & $112(57.7)$ & $74(62.7)$ & 91 (77.1) & \\
\hline Lymph node status & & & & 0.56 \\
\hline 0 & $52(26.8)$ & 31 & 43 & \\
\hline $1-3$ & 98 (50.5) & 57 & 30 & \\
\hline 4 & 43 (22.2) & 50 & 25 & \\
\hline Not registered & $1(0.05)$ & & & \\
\hline NHG & & & & $<0.001$ \\
\hline I & 44 (22.7) & $6(5.1)$ & $0(0)$ & \\
\hline ॥ & $104(53.6)$ & $48(40.7)$ & $19(16.1)$ & \\
\hline III & $42(21.6)$ & $58(49.1)$ & $94(79.7)$ & \\
\hline Not evaluated & $4(2.1)$ & $6(5.1)$ & $5(4.2)$ & \\
\hline Mitotic index & & & & \\
\hline 1 & $99(51.0)$ & $27(22.9)$ & $7(5.9)$ & $<0.001$ \\
\hline 2 & 63 (32.5) & 49 (41.5) & $30(25.4)$ & \\
\hline 3 & $28(14.4)$ & $36(30.5)$ & $76(64.4)$ & \\
\hline Not evaluated & $4(2.1)$ & $6(5.1)$ & $5(4.2)$ & \\
\hline Oestrogen receptor & & & & $<0.001$ \\
\hline Negative & $33(17.0)$ & $34(28.8)$ & $71(60.2)$ & \\
\hline Positive & $155(79.9)$ & $81(68.6)$ & $44(37.3)$ & \\
\hline Not evaluated & $6(3.1)$ & $3(2.5)$ & $3(2.5)$ & \\
\hline $\begin{array}{l}\text { Progesterone } \\
\text { receptor }\end{array}$ & & & & $<0.001$ \\
\hline Negative & $35(18.0)$ & $41(34.7)$ & $76(64.4)$ & \\
\hline Positive & $147(75.8)$ & $68(57.6)$ & 37 (31.3) & \\
\hline Not evaluated & $12(6.2)$ & $9(7.6)$ & $5(4.2)$ & \\
\hline
\end{tabular}

The $p$ values were assessed by the $\chi^{2}$ test.

NHG, Nottingham histological grade.

Table 2 ER positivity in relation to $\mathrm{Ki}-67$ index in the two study groups

\begin{tabular}{llllll}
\hline \multicolumn{7}{c}{ Ki-67 index } \\
\cline { 2 - 6 } & $\mathbf{0 - 1 \%}$ & $\mathbf{2 - 1 0 \%}$ & $\mathbf{1 1 - 2 5 \%}$ & $\mathbf{2 6 - 5 0 \%}$ & $\mathbf{5 1 - 1 0 0 \%}$ \\
\hline $\begin{array}{l}\text { Number ER positive } \\
\text { Tamoxifen } v \text { control* }\end{array}$ & 42 & 113 & 81 & 37 & 7 \\
\hline *Log rank recurrence free survival p value. & 0.05 & 0.03 & 0.66 & 0.02 & 0.07 \\
ER, oestrogen receptor.
\end{tabular}


Our study was approved by the ethical committees at Linköping and Lund Universities, Sweden.

\section{Tissue array and immunohistochemistry}

Areas representative of invasive cancer were marked on the haematoxylin and eosin stained slides and two separate tissue microarrays were constructed, one using a manual (MTA-1) and the other an automated (ATA-27) arrayer (both from Beecher Inc, Sun Prairie, Wisconsin, USA). Two $0.6 \mathrm{~mm}$ tissue cores were taken from each donor block and mounted in a recipient block. There were approximately 200 tissue cores in each recipient block. Cores were generally taken from the peripheral part of the tumour in cases where the tumour had relatively well defined borders. For technical reasons, in more diffusely growing tumours, areas with the highest tumour cell density were primarily targeted. Sections $(4 \mu \mathrm{m}$ thick) were dried, dewaxed, rehydrated, and microwave treated for $2 \times 5$ minutes in citrate buffer $(\mathrm{pH} 6.0)$ before being processed in an automatic immunohistochemistry staining machine (Techmate 500; Dako, Copenhagen, Denmark) for Ki-67, using an anti-Ki-67 monoclonal antibody (1/200 dilution; M7240; Dako). For immunohistochemical evaluation of Ki-67, a scoring system based on the estimated fractions of positively staining nuclei was used as follows: $0,0-1 \%$; $1,1-10 \% ; 2,11-25 \% ; 3,26-50 \%$; and $4,51-$ $100 \%$. The intensity of the nuclear staining for Ki-67 varied slightly, but was distinct in most cases.

In addition, the tissue microarrays were analysed immunohistochemically for ER and PR status using the Ventana Benchmark system (Ventana Medical Systems Inc, Tucson, Arizona, USA), with prediluted antibodies (anti-ER clone 6Fll and anti-PR clone 16). The fractions of ER and PR positive nuclei were determined and classified into four groups $(0-10 \%, 11-50 \%, 51-75 \%$, and $>75 \%)$. In line with the clinically established cutoff value used for hormone receptor assessment, tumours with more than $10 \%$ positively staining nuclei were considered positive. The MI had previously been assessed along with histological grading, by counting the number of mitoses/10 high power fields and adjusting for the optics used..$^{30}$ Tumours had then been given a score from 1 (low) to 3 (high). For this investigation, an additional reevaluation regarding histological type ${ }^{31}$ was performed.

\section{Statistics}

Differences in distribution between clinical data and tumour characteristics were analysed by the $\chi^{2}$ test. RFS, BCS, and overall survival OS were estimated according to the KaplanMeier method and the log rank test was used to compare survival in different strata. RFS considered local, regional, and distant recurrences and breast cancer specific death, but not contralateral breast cancer, as a primary event. Causes of death were obtained from Statistics Sweden. For BCS, patients who died of any other cause were censored at the time of death. A Cox proportional hazards model was used for the estimation of the relative risk in univariate and multivariate analyses. The interaction between tamoxifen treatment and the investigated parameters was further explored by Cox models including one of the four variables respectively, a treatment variable and an interaction variable. All statistical tests were two sided and the calculations were performed with SPSS 11.0 (SPSS Inc, Chicago, Illinois, USA).

\section{RESULTS}

\section{Tumour type and hormone receptor status}

Paraffin wax blocks were available from 500 patients included in the randomised treatment trial and assessment of histological type was possible in 493 cases. IDCs formed the largest group (83\%), followed by ILCs $(8.7 \%)$. There was
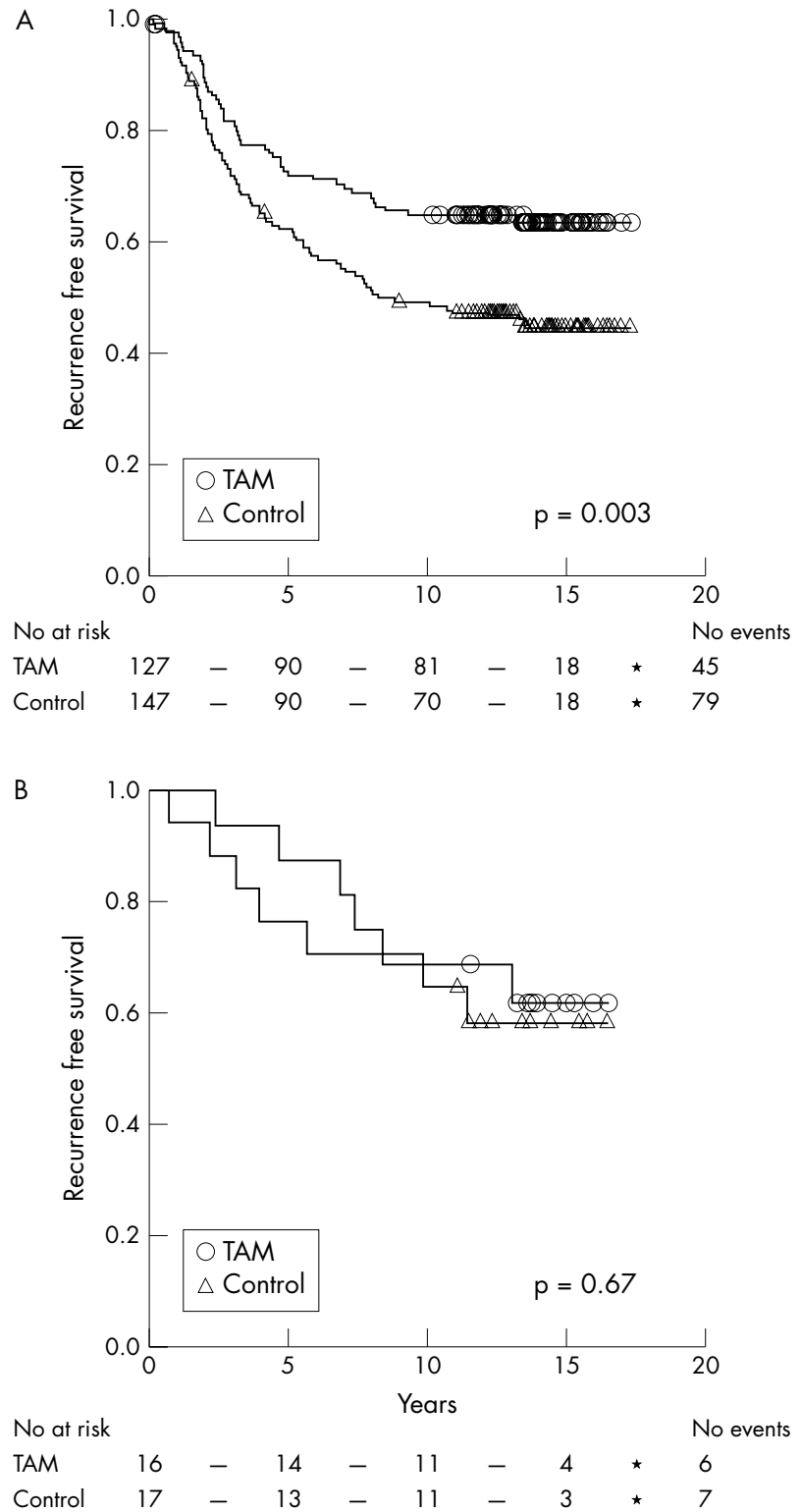

Figure 1 Kaplan-Meier estimates of recurrence free survival according to treatment arm for oestrogen receptor positive cases of $(A)$ invasive ductal carcinomas $(n=274)$ and $(B)$ invasive lobular carcinoma ( $n=33$ ). TAM, tamoxifen treated.

no significant difference in the histological type distribution between patients randomised to tamoxifen treatment or no treatment. Table l summarises the clinicopathological characteristics of the histological subgroups of IDC and ILC. In general, a larger proportion of ILCs were hormone receptor positive compared with IDC. In addition, most ILCs were strongly ER and PR positive, with $>75 \%$ of the nuclei being positive.

\section{Proliferation markers and histological grade}

Immunohistochemical Ki-67 expression could be evaluated in 430 cases $(86 \%)$. Nottingham histological grade (NHG) including MI had previously been evaluated in 514 cases with the following distribution for MI: 1,170 cases $(33.1 \%) ; 2,171$ cases (33.3\%); 3, 173 (33.6\%); and for NHG: I, 58 (11.3\%); II, $222(43.2 \%)$; and III, $234(45.5 \%)$. The fraction of Ki-67 positive cells correlated significantly with MI $(\mathrm{p}<0.0001)$. In addition, in the 286 tumours that had previously been 
Table 4 RFS and BCS by Cox univariate analysis in ER positive tumors

\begin{tabular}{|c|c|c|c|c|c|c|}
\hline \multirow[b]{2}{*}{ Category } & \multicolumn{3}{|l|}{ RFS } & \multicolumn{3}{|l|}{ BCS } \\
\hline & RR & $95 \% \mathrm{Cl}$ & $\mathrm{p}$ Value & RR & $95 \% \mathrm{Cl}$ & $p$ Value \\
\hline \multicolumn{7}{|l|}{ IDC } \\
\hline Control & 1.00 & & & 1.00 & & \\
\hline Tamoxifen & 0.58 & 0.40 to 0.83 & 0.003 & 0.71 & 0.48 to 1.07 & 0.10 \\
\hline \multicolumn{7}{|l|}{ ILC } \\
\hline Control & 1.00 & & & 1.00 & & \\
\hline Tamoxifen & 0.79 & 0.26 to 2.36 & 0.67 & 0.91 & 0.28 to 2.99 & 0.88 \\
\hline \multicolumn{7}{|c|}{ Low $\mathrm{Ki}-67$ index } \\
\hline Control & 1.00 & & & 1.00 & & \\
\hline Tamoxifen & 0.50 & 0.29 to 0.87 & 0.01 & 0.68 & 0.37 to 1.27 & 0.22 \\
\hline \multicolumn{7}{|c|}{ Intermediate Ki-67 index } \\
\hline Control & 1.00 & & & 1.00 & & \\
\hline Tamoxifen & 1.15 & 0.61 to 2.17 & 0.66 & 1.40 & 0.69 to 2.71 & 0.37 \\
\hline \multicolumn{7}{|c|}{ High Ki-67 index } \\
\hline Control & 1.00 & & & 1.00 & & \\
\hline Tamoxifen & 0.29 & 0.11 to 0.74 & 0.006 & 0.38 & 0.15 to 0.97 & 0.04 \\
\hline \multicolumn{7}{|l|}{$\mathrm{Ml}$ of 1} \\
\hline Control & 1.00 & & & 1.00 & & \\
\hline Tamoxifen & 0.63 & 0.34 to 1.16 & 0.14 & 0.57 & 0.28 to 1.12 & 0.13 \\
\hline \multicolumn{3}{|l|}{$\mathrm{Ml}$ of 2} & & & & \\
\hline Tamoxifen & 0.70 & 0.41 to 1.19 & 0.18 & 1.01 & 0.57 to 1.80 & 0.96 \\
\hline \multicolumn{7}{|l|}{$\mathrm{Ml}$ of 3} \\
\hline Control & 1.00 & & & 1.00 & & \\
\hline Tamoxifen & 0.50 & 0.26 to 0.99 & 0.04 & 0.62 & 0.31 to 1.22 & 0.16 \\
\hline \multicolumn{7}{|c|}{ Grade I tumours } \\
\hline Control & 1.00 & & & 1.00 & & \\
\hline Tamoxifen & 0.58 & 0.18 to 1.89 & 0.37 & 0.23 & 0.03 to 1.94 & 0.18 \\
\hline \multicolumn{7}{|c|}{ Grade II tumours } \\
\hline Control & 1.00 & & & 1.00 & & \\
\hline Tamoxifen & 0.63 & 0.39 to 1.01 & 0.06 & 0.82 & 0.49 to 1.40 & 0.47 \\
\hline \multicolumn{7}{|c|}{ Grade III tumours } \\
\hline Control & 1.00 & & & 1.00 & & \\
\hline Tamoxifen & 0.50 & 0.29 to 0.87 & 0.01 & 0.59 & 0.34 to 1.04 & 0.07 \\
\hline
\end{tabular}

analysed by flow cytometry, ${ }^{15}$ there was a significant correlation between Ki-67 and $S$ phase fraction $(\mathrm{p}<0.0001$; data not shown). Tables 2 and 3 show the distribution of clinicopathological parameters in the different Ki-67 groups. ER status was available for 457 cases.

\section{Survival analyses regarding tamoxifen response}

For survival analyses, all ER positive $(>10 \%)$ cases were included, irrespective of PR status, which is line with the current recommendation regarding endocrine treatment. As described previously, ${ }^{29}$ patients with ER positive tumours receiving two years of tamoxifen had a significantly higher RFS $(p=0.005)$. The increase in BCS was not significant $(\mathrm{p}=0.13)$, although there was a more favourable trend in the tamoxifen treated group. Tamoxifen treatment had no effect in receptor negative tumours.

We next delineated the tamoxifen response in different histological subgroups of breast cancer. Tamoxifen treatment was associated with a significantly increased RFS for ER positive IDC ( $p=0.003$ ), whereas there was no significant difference in RFS for ILC $(p=0.67)$ (fig 1). Tamoxifen treatment also improved BCS in IDC, although it did not reach significance $(p=0.10)$. The Kaplan-Meier plot for ILC shows slightly diverging curves in favour of the tamoxifen treated group early during treatment, but the lines converged later. Thus, after five years, RFS was $72 \%$ versus $62 \%$ and $87 \%$ versus $76 \%$ for treated and untreated patients with IDC and ILC, respectively. After 10 years, RFS was $65 \%$ versus $49 \%$ and $68 \%$ versus $65 \%$ in IDC and ILC, respectively, with similar proportions at 15 years. Interestingly, overall survival after 10 years, which was similar to BCS, was $76 \%$ versus $68 \%$ and $60 \%$ versus $69 \%$ for untreated and treated patients with ILC and IDC, respectively.
In Cox univariate analyses, the relative risk of disease recurrence after tamoxifen treatment was 0.79 and 0.58 for ILC and IDC, respectively, whereas BCS was 0.91 and 0.71 respectively, with a large confidence interval for ILC, because of the relatively small number of tumours (table 4).

Initially, we characterised the tamoxifen response in the five different Ki-67 proliferation groups (table 2); there was a significant response in the two lowest groups and in the two highest groups, whereas there was no difference in survival between untreated patients and tamoxifen treated patients in the intermediate group. Therefore, in the following analyses we divided the material into three groups corresponding to: $0-10 \%$ (low), $11-25 \%$ (intermediate), and $>25 \%$ (high) expression (table 3). Using this subdivision, RFS was significantly increased in the Ki-67 low $(p=0.01)$ and high $(p=0.006)$ groups, whereas tumours in the intermediate group did not show a significant $(\mathrm{p}=0.66)$ tamoxifen response (fig 2). BCS (fig 3 ) was significantly improved by tamoxifen treatment in the group with high Ki-67 expression $(p=0.04)$, but not in the low $(p=0.22)$ and intermediate $(\mathrm{p}=0.37)$ groups. In the group with intermediate Ki-67 expression, there was a tendency towards a shorter BCS after tamoxifen treatment (fig 3B).

The MI is one of three components of the NHG and we therefore characterised the tamoxifen response in low, intermediate, and high proliferative tumours defined by the mitotic count. The trend was similar to that seen for Ki-67 with regard to RFS and BCS, although it was not as pronounced (table 4). The only significant difference in RFS was seen in the group with the highest MI $(p=0.04)$. In contrast, and in line with the Ki-67 data, BCS was not improved by tamoxifen treatment in the intermediate group $(p=0.96)$, compared with a more obviously positive, 
A

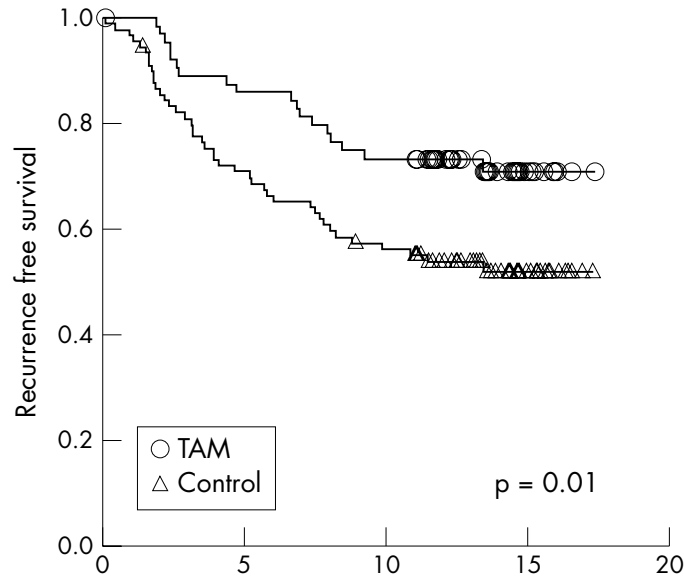

No at risk

TAM $65-55-47-9$ - 65 18

Control $90-63-49-15$ * 42

B

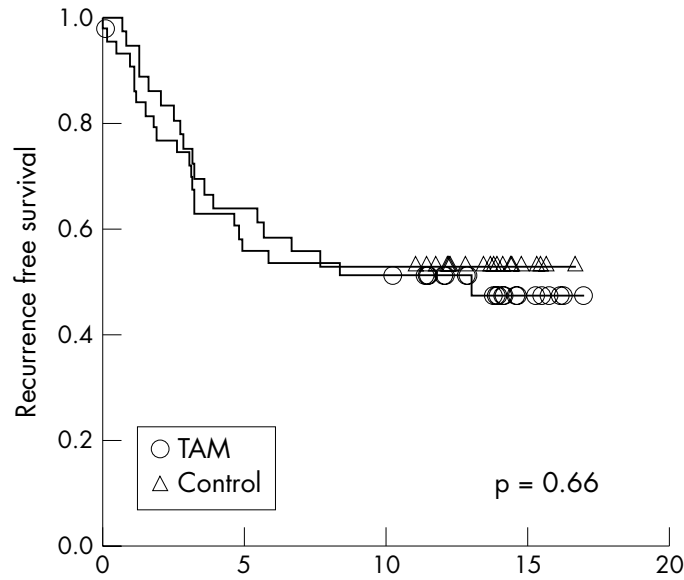

No at risk

TAM $44-24-22-6$ * 22

Control $36-23-19-4$ * 17

C

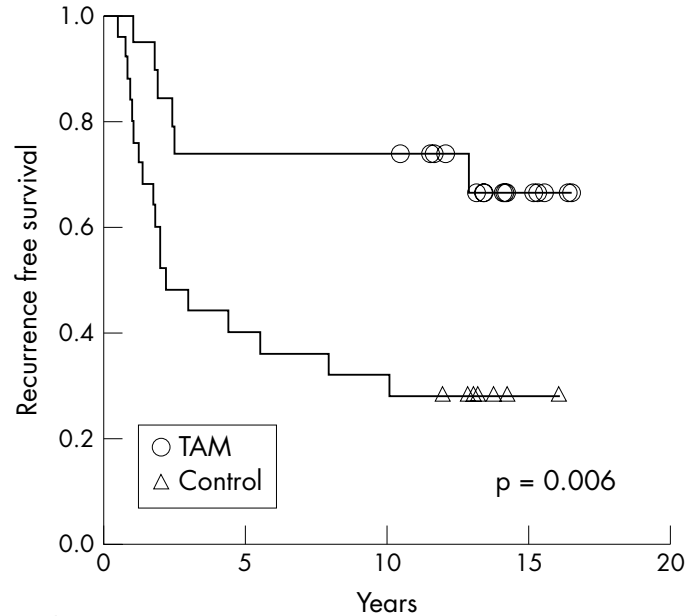

No at risk

$\begin{array}{llllllllll}\text { TAM } & 19 & - & 14 & - & 14 & - & 5 & \star & 6 \\ \text { Control } & 25 & - & 10 & - & 8 & - & 1 & \star & 18\end{array}$

Figure 2 Kaplan-Meier estimates of recurrence free survival according to treatment arm for oestrogen receptor positive cases with (A) low, (B) intermediate, and (C) high Ki-67 expression. TAM, tamoxifen treated.
A

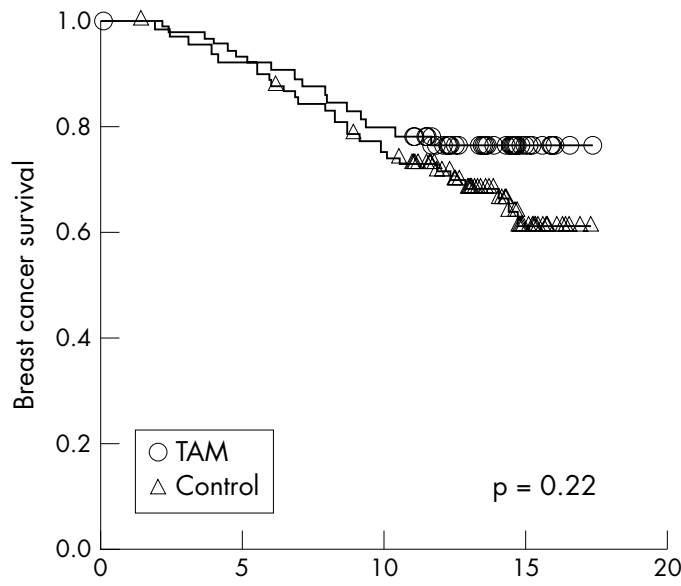

No at risk No events TAM $65-59-51-9 \star 15$ Control $90-83-65-17$ * 30

B

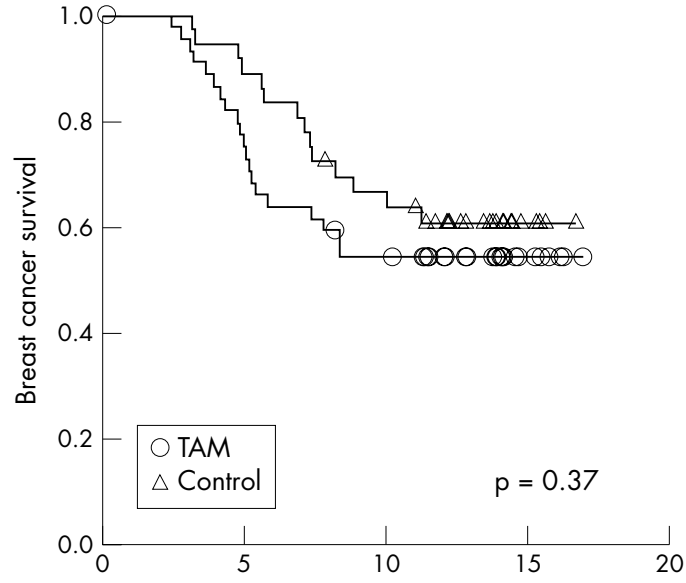

No at risk No events TAM $45-33-23-6$ - 20 Control $36-32-23-4 \star 14$

C

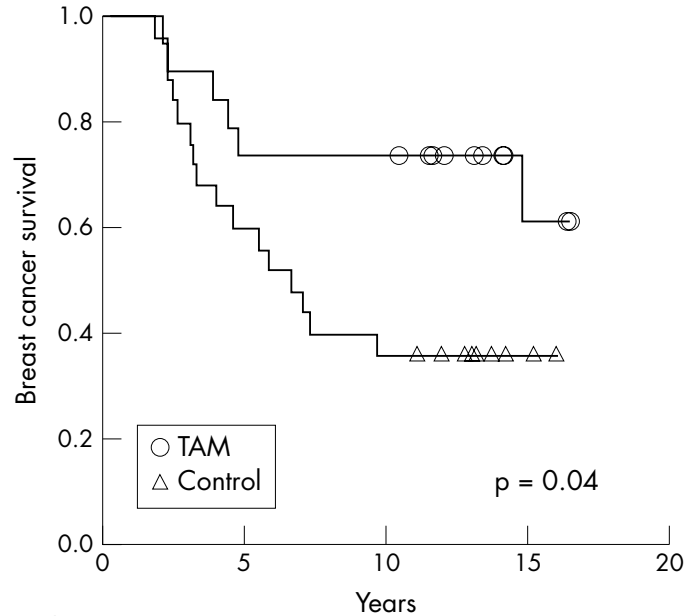

No at risk

Years

No events

$\begin{array}{lrlrlrllll}\text { TAM } & 19 & - & 14 & - & 14 & - & 5 & \star & 6 \\ \text { Control } & 25 & - & 15 & - & 9 & - & 2 & \star & 16\end{array}$

Figure 3 Kaplan-Meier estimates of breast cancer survival according to treatment arm for oestrogen receptor positive cases with (A) low, (B) intermediate, and (C) high Ki-67 expression. TAM, tamoxifen treated. 
although not significant, tamoxifen effect in the low $(\mathrm{p}=0.13)$ and high $(\mathrm{p}=0.16)$ groups.

Regarding NHG, RFS was significantly improved for grade II and III tumours ( $p=0.06$ and $p=0.01$, respectively), but not for the relatively few grade I tumours ( $p=0.37$ ). There was a borderline significant increase in BFS after tamoxifen treatment in grade III tumours $(p=0.07)$, but not in grade I and II tumours (table 4).

The interaction between tamoxifen treatment and histological type, Ki-67, grade, and MI was further explored by a Cox model including one of the four variables, a treatment variable and an interaction variable. Using this approach, a significant interaction was found between intermediate compared with low and high Ki-67 expression and tamoxifen treatment, even when adjusted for other important clinicopathological variables (table 5). None of the other variables, including histological type, showed a significant interaction with tamoxifen using this approach.

\section{Survival analyses in patients not receiving adjuvant tamoxifen}

In total, 288 patients were allocated to the control arm, which formed an untreated patient group suitable for studies of prognostic information without interference from adjuvant medication. Table 6 provides an overview of the prognostic impact of the factors investigated in our study. In the untreated group, proliferation markers in general showed a linear association with clinical outcome, with the best clinical outcome for tumours with low proliferation and worst for highly proliferating tumours.

\section{DISCUSSION}

Our study provides an ideal setting to study the endocrine response, because all patients had been randomised to tamoxifen as monotherapy, or to the control arm. Furthermore, there were no differences in the clinicopathological variables between the trial arms or different histological tumour types that could influence outcome. However, it should be noted that upon disease recurrence, tamoxifen treatment was also offered to patients initially randomised to the control group. This fact might interfere with the validity of the analyses of BCS or overall survival, but not RFS.

Histological tumour type appeared to be a predictor of tamoxifen response, with a highly significant survival benefit for RFS and almost significant benefit for BCS in IDC, in contrast to the small subset of ILCs, which did not benefit significantly from treatment. However, in multivariate interaction analyses, no significantly different tamoxifen response could be demonstrated in IDC compared with ILC. Larger studies or metaanalyses are needed to establish such an association with acceptable statistical power.

In general, ILC shows a more diffuse, infiltrative growth pattern and is more often multifocal than IDC. However, this was not reflected by the number of local recurrences-four of $43(9 \%)$ in ILC and 38 of $411(9 \%)$ in IDC. Node positivity was also similar for ILC and IDC. It is clear that ILCs have different biological characteristics to IDCs, which are mirrored by specific genetic alterations in this subgroup of tumours. ${ }^{32}{ }^{33}$ The increasing incidence of ILC during the past decade $^{3435}$ further stresses the importance of clarifying the effect of tamoxifen treatment in this breast cancer subgroup.

"The traditional morphological examination, including evaluation of proliferation, seems to mirror the differential expression of hundreds of genes clearly linked to the clinical behaviour of tumours"

We also investigated the relation between the tamoxifen response and two proliferation markers: Ki-67 expression by immunohistochemistry and MI. The MI is routinely assessed as one of the three cornerstones of the Elston Ellis histological grading system (NHG). Interestingly, irrespective of the method used for assessing proliferative activity, the beneficial effect of tamoxifen was significant in highly proliferating tumours, both in terms of RFS and BCS, suggesting an important role for tamoxifen in this group of tumours. In contrast, those tumours with neither high nor low proliferation showed a worse response to tamoxifen. This was most apparent for Ki-67, where the high and low groups showed a significant response, whereas the intermediate group showed no response. The difference was significant for RFS and BCS, even in interaction analyses. When proliferation was defined by the MI, only highly proliferating tumours showed a significant tamoxifen response with regard to RFS, whereas the effect on BCS was similar to that seen for Ki-67, with an impaired tamoxifen response in the intermediate group. The reason why intermediately proliferating ER positive tumours have an impaired tamoxifen response is unclear. Although the choice of cutoff levels for immunohistochemical analyses, in this case Ki-67, may be disputed, our results indicate divergent behaviour for the intermediately proliferating group after tamoxifen treatment. Although this pattern may be coincidental, it must be compared with the

Table 5 Multivariate Cox proportional hazards model for Ki-67 index and treatment interaction for ER positive tumours*

\begin{tabular}{|c|c|c|c|c|c|c|c|}
\hline \multirow[b]{2}{*}{ Variable } & & \multicolumn{3}{|l|}{ RFS } & \multicolumn{3}{|l|}{ BCS } \\
\hline & & $\mathbf{R R}$ & $95 \% \mathrm{Cl}$ & p Value & $\mathbf{R R}$ & $95 \% \mathrm{Cl}$ & p Value \\
\hline $\mathrm{Ki}-67$ & Intermediate $v$ low + high & 0.85 & 0.50 to 1.46 & 0.56 & 0.81 & 0.51 to 1.69 & 0.93 \\
\hline Treatment & Tamoxifen $v$ control & 0.46 & 0.29 to 0.74 & 0.001 & 0.61 & 0.36 to 1.02 & 0.06 \\
\hline Interaction variable & Tamoxifen $\times \mathrm{Ki}-67$ & 2.63 & 1.19 to 5.82 & 0.02 & 2.38 & 1.01 to 5.63 & 0.05 \\
\hline $\mathrm{Ml}$ & $2 \vee 1+3$ & 1.22 & 0.80 to 1.88 & 0.35 & 1.04 & 0.64 to 1.69 & 0.88 \\
\hline Treatment & Tamoxifen $v$ control & 0.60 & 0.38 to 0.94 & 0.02 & 0.66 & 0.40 to 1.09 & 0.10 \\
\hline Interaction variable & Tamoxifen $\times$ Ml & 1.18 & 0.59 to 2.36 & 0.64 & 1.59 & 0.74 to 3.39 & 0.23 \\
\hline NHG & $3 \vee 1+2$ & 1.86 & 1.17 to 2.95 & 0.008 & 2.43 & 1.46 to 4.06 & 0.001 \\
\hline Treatment & Tamoxifen $v$ control & 0.62 & 0.40 to 0.96 & 0.03 & 0.76 & 0.45 to 1.25 & 0.28 \\
\hline Interaction variable & Tamoxifen $\times$ NHG & 0.94 & 0.46 to 1.92 & 0.87 & 0.96 & 0.44 to 2.05 & 0.91 \\
\hline Histological type & ILC $v$ IDC & 0.70 & 0.32 to 1.55 & 0.38 & 0.88 & 0.37 to 2.09 & 0.78 \\
\hline Treatment & Tamoxifen $v$ control & 0.59 & 0.40 to 0.85 & 0.005 & 0.75 & 0.50 to 1.13 & 0.17 \\
\hline Interaction variable & Tamoxifen $\times$ type & 1.39 & 0.44 to 4.39 & 0.58 & 1.17 & 0.33 to 4.09 & 0.81 \\
\hline
\end{tabular}

*Adjusted for age (continuous), tumour size (T1 $v \mathrm{~T} 2)$, and nodal status ( $\mathrm{NO} v \mathrm{~N}+$ ).

$\mathrm{BCS}$, breast cancer survival; Cl, confidence interval; IDC, invasive ductal carcinomas; ILC, invasive lobular carcinoma; MI, mitotic index; NHG, Nottingham histological grade; RFS, recurrence free survival; RR, relative risk. 
Table 6 Univariate analyses of RFS and BCS in patients without adjuvant tamoxifen treatment

\begin{tabular}{|c|c|c|c|c|c|c|c|}
\hline \multirow[b]{2}{*}{ Variable } & & \multicolumn{3}{|l|}{ RFS } & \multicolumn{3}{|l|}{ BCS } \\
\hline & & RR & $95 \% \mathrm{Cl}$ & p Value & RR & $95 \% \mathrm{Cl}$ & p Value \\
\hline Tumour type & ILC $v$ IDC & 0.63 & 0.32 to 1.24 & 0.17 & 0.71 & 0.34 to 1.46 & 0.35 \\
\hline $\mathrm{Ki}-67$ & $3 v 1+2$ & 1.58 & 1.06 to 2.35 & 0.03 & 1.90 & 1.24 to 2.92 & 0.003 \\
\hline Mitotic index & $2+3 \times 1$ & 1.98 & 1.34 to 2.92 & 0.01 & 2.06 & 1.32 to 3.19 & 0.001 \\
\hline NHG & $3 \vee 1+2$ & 1.82 & 1.30 to 2.54 & $<0.001$ & 2.42 & 1.67 to 3.53 & $<0.001$ \\
\hline Age & Continuous & 0.97 & 0.94 to 0.99 & 0.009 & 0.96 & 0.93 to 0.99 & 0.006 \\
\hline Node status & $\mathrm{N}+v \mathrm{NO}$ & 1.97 & 1.29 to 3.02 & $<0.01$ & 2.21 & 1.35 to 3.61 & $<0.01$ \\
\hline Tumour size & $\mathrm{T} 2$ v T1 & 1.24 & 0.89 to 1.73 & 0.21 & 1.40 & 0.96 to 2.03 & 0.08 \\
\hline
\end{tabular}

BCS, breast cancer survival; Cl, confidence interval; IDC, invasive ductal carcinomas; ILC, invasive lobular carcinoma; NHG, Nottingham histological grade; RFS, recurrence free survival; RR, relative risk.

findings in the untreated arm, where a linearly inverse relation was seen between tumour proliferation and prognosis. Although there were few ILCs in this group (table 1), tumours with other phenotypic alterations associated with tamoxifen resistance, such as HER2, EGFR, and cyclin D1 overexpression might be clustered in the intermediately proliferating group, and future studies are needed to delineate these potential links.

Our study investigated tumours with more than 10\% ER positive cells, irrespective of PR status. However, similar findings were obtained when analysing ER and/or PR positive tumours ( $3 \%$ were PR positive/ER negative), supporting the notion that the observations were not dependent on PR status (data not shown). In addition, similar results were obtained when tumours with more than $75 \%$ ER positive cells were analysed separately, further suggesting that the difference in treatment response was not the result of variations in the absolute fraction of ER positive cells.

Although data from molecular profiling assays may add important information regarding treatment predictive and prognostic variables, the traditional morphological examination, including evaluation of proliferation, seems to mirror the differential expression of hundreds of genes clearly linked to the clinical behaviour of tumours.

In summary, we investigated the relation between established histopathological parameters and tamoxifen response in premenopausal women included in a randomised trial, and found links between proliferation and tamoxifen response. In addition to hormone receptor status, these parameters seem to provide important information and will probably remain essential (at least to some extent) in the future tailoring of adjuvant breast cancer treatment. We found a potentially impaired tamoxifen response in the relatively few lobular carcinomas studied, but larger studies are needed to investigate this highly relevant issue.

\section{Take home messages}

- We investigated the relation between established histopathological parameters and tamoxifen response in premenopausal women included in a randomised trial, and found links between proliferation and tamoxifen response

- In general, highly and lowly proliferating tumours showed a better response to tamoxifen treatment than intermediately proliferating tumours

- The tamoxifen response appeared to be impaired in the relatively few lobular carcinomas studied, but larger studies are needed to investigate this issue

\section{ACKNOWLEDGEMENTS}

We are grateful for excellent technical assistance from E Nilsson. This study was supported by grants from the Swedish Cancer Society, Swegene/Wallenberg Consortium North, Gunnar, Arvid and Elisabeth Nilsson Cancer Foundation, Per-Eric and Ulla Schyberg Foundation, Lund University Research Funds, and Malmö University Hospital Research and Cancer Funds.

\section{Authors' affiliations}

K Jirström, L Rydén, L Anagnostaki, G Landberg, Division of Pathology Department of Laboratory Medicine, Lund University, Malmö University Hospital, SE-205 02, Malmö, Sweden

B Nordenskiöld, O Stål, Department of Biomedicine and Surgery, Division of Oncology, Linköping University, SE-581 83 Linköping, Sweden

S Thorstenson, Department of Pathology and Cytology, Kalmar County Hospital, SE-391 85 Kalmar, Sweden

G Chebil, Department of Pathology, Helsingborg Hospital, SE-251 87 Helsingborg, Sweden

P-E Jönsson, Department of Surgery, Helsingborg Hospital

M Fernö, Department of Oncology, Lund University Hospital, SE-221 85 Lund, Sweden

On behalf of the South Swedish and South-East Swedish breast cancer groups.

\section{REFERENCES}

1 Early Breast Cancer Trialist's Collaborative Group. Tamoxifen for early breast cancer: an overview of the randomised trials. Lancet 1998;351:1451-67.

2 Ferno M, Baldetorp B, Hatschek T, et al. Results of two or five years of adjuvant tamoxifen correlated to steroid receptor and S-phase levels. Breast Cancer Res Treat 2000;59:69-76.

3 Bryant J, Fisher B, Dignam J. Duration of adjuvant tamoxifen therapy. J Natl Cancer Inst 2001;30:56-61.

4 Stewart $H$, Prescott $R$, Forrest P. Scottish adjuvant tamoxifen trial: a randomised study updated to 15 years. J Natl Cancer Inst 2001;93:456-62.

5 Swedish Breast Cancer Cooperative Group. Randomized trial of two versus five years of adjuvant tamoxifen for postmenopausal early stage breast cancer. J Natl Cancer Inst 1996;88:1543-9.

6 Elledge RM, Green S, Pugh R, et al. Estrogen receptor (ER) and progesterone receptor (PgR), by ligand-binding assay compared with $E R, P g R$ and $p S 2$, by immuno-histochemistry in predicting response to tamoxifen in metastatic breast cancer: a southwest oncology group study. Int J Cancer 2000;89:111-17.

7 Bardou V, Arpino G, Elledge R, et al. Progesterone receptor status significantly improves outcome prediction over estrogen receptor status alone for adjuvant endocrine therapy in two large databases. J Clin Oncol 2003;21:1973-9.

8 Borg A, Baldetorp B, Fernö M, et al. ERBB2 amplification is associated with tamoxifen resistance in steroid-receptor positive breast cancer. Cancer Lett 1994;81:137-44.

9 Stal O, Borg A, Ferno M, et al. ErbB2 status and the benefit from two or five years of adjuvant tamoxifen in postmenopausal early stage breast cancer. Ann Oncol 2000; 11:1545-50.

10 De Placido S, De Laurentiis M, Carlomagno C, et al. Twenty-year results of the Naples GUN randomized trial: predictive factors of adjuvant tamoxifen efficacy in early breast cancer. Clin Cancer Res 2003;9:1039-46.

11 Grogan M, Tabar L, Chua B, et al. Estimating the benefits of adjuvant systemic therapy for women with early breast cancer. Br J Surg 2001;88:1513-18.

12 Smith D, Howell A, Wagstaff J. Infiltrating lobular carcinoma of the breast: response to endocrine therapy and survival. Eur J Cancer Clin Oncol 1987;23:979-82. 
13 Mathieu M, Rouzier R, Llombart-Cussac A, et al. The poor responsiveness of infiltrating lobular breast carcinoma to neoadjuvant chemotherapy can be explained by their biological profile. Eur J Cancer 2004;40:342-51.

14 Isola J, Helin $\mathrm{H}$, Helle $M$, et al. Evaluation of cell proliferation in breast carcinoma. Comparison of Ki-67 immunohistochemical study, DNA flow cytometric analysis and mitotic count. Cancer 1990;65:1 180-4.

15 Ferno M, Baldetorp B, Bendahl P-O, et al. Recurrence-free survival in breas cancer improved by adjuvant tamoxifen-especially for progesterone receptor positive tumors with a high proliferation. Breast Cancer Res Treat 1995;36:23-34.

16 Keshgegian A, Cnaan A. Proliferation markers in breast carcinoma. Mitotic figure count, S-phase fraction, proliferating cell nuclear antigen, $\mathrm{Ki}-67$ and MIB-1. Am J Clin Pathol 1995; 104:42-9.

17 Michels J-J, Duigou F, Marnay J. Flow cytometry in primary breast carcinomas. Prognostic impact of proliferative activity. Breast Cancer Res Treat 2000;62:117-26.

18 Kallioniemi O-P, Hietanen T, Mattila J, et al. Aneuploid DNA content and high $\mathrm{S}$-phase fraction of tumour cells are related to poor prognosis in patients with primary breast cancer. Eur J Cancer Clin Oncol 1987:23:277-82.

19 Stal O, Skoog L, Rutqvist L, et al. S-phase fraction and survival benefit from adjuvant chemotherapy or radiotherapy of breast cancer. $\mathrm{Br} J$ Cancer 1994;70:1258-62.

20 Faneyte I, Schrama J, Peterse J, et al. Breast cancer response to neoadjuvant chemotherapy: predictive markers and relation with outcome. $\mathrm{Br} J$ Cancer 2003;88:406-12.

21 Aas T, Geisler S, Eide G, et al. Predictive value of tumour cell proliferation in locally advanced breast cancer treated with neoadjuvant chemotherapy. Eur J Cancer 2003;39:438-46.

22 Petit T, Wilt $M$, Velten $M$, et al. Comparative value of tumour grade, hormonal receptors, Ki-67, HER-2 and topoisomerase II alpha status as predictive markers in breast cancer treated with neoadjuvant anthracyclin-based chemotherapy. Eur J Cancer 2004;40:205-11.

23 Remvikos $Y$, Beuzeboc $\mathrm{P}$, Zajdela $\mathrm{A}$, et al. Correlation of pretreatment proliferative activity of breast cancer with the response to cytotoxic chemotherapy. J Natl Cancer Inst 1989;81:1383-7.
24 Silvestrini R, Daidone M, Mastore $M$, et al. Cell kinetics as a predictive factor in node-positive breast cancer treated with adjuvant hormone therapy. J Clin Oncol 1993; 11:1150-5.

25 Daidone M, Luisi A, Martelli E, et al. Biomarkers and outcome after tamoxifen treatment in node-positive breast cancers from elderly women. $\mathrm{Br} J$ Cancer 2000:82:270-7.

26 Nicholson R, Bouzubar N, Walker K, et al. Hormone sensitivity in breast cancer: influence of heterogeneity of oestrogen receptor expression and cell proliferation. Eur J Cancer 1991;27:908-13.

27 Paradiso A, Tommasi S, Mangia A, et al. Tumor-proliferative activity, progesterone receptor status, estrogen receptor level, and clinical outcome of estrogen receptor-positive advanced breast cancer. Cancer Res 1990;50:2958-62.

28 International Union against Cancer (UICC). TNM classification of malignant tumours. Berlin-Heidelberg-New York: Springer-Verlag, 1987.

29 Rydén L, Jönsson P-E, Chebil G, et al. Two years of adjuvant tamoxifen in premenopausal patients with breast cancer: a randomised, controlled trial with long-term follow-up. Eur J Cancer 2005;2:256-64

30 Elston CW, Ellis IO. Pathological prognostic factors in breast cancer. 1. The value of histological grade in breast cancer: experience from a large study with long-term follow-up, Histopathology 1991;19:403-10.

31 WHO. International histological classification of tumours. Histologic types of breast tumours. Geneva: World Health Organisation, 1991.

32 Gunther K, Merkelbach-Bruse S, Amo-Takyi B, et al. Differences in genetic alterations between primary lobular and ductal breast cancers detected by comparative genomic hybridization. J Pathol 2001;193:40-7.

33 Korkola J, DeVries S, Fridlyand J, et al. Differentiation of lobular versus ductal breast carcinomas by expression microarray analysis. Cancer Res 2003:63:7167-75

34 Li C, Weiss N, Stanford J, et al. Hormone replacement therapy in relation to risk of lobular and ductal breast carcinoma in middle-aged women. Cancer 2000;88:2570-7.

35 Verkooijen H, Fioretta G, Vlastos $G$, et al. Important increase of invasive lobular breast cancer incidence in Geneva, Switzerland. Int J Cancer 2003;104:778-81. 